\title{
Simultanious Localization, MAPING AND SCENE RECONSTRUCTION
}

\author{
LEPEJ, P., LAKOTA, M., RAKUN, J.
}

Abstract: This work describes an approach for simultaneous localization and mapping as well as $3 D$ reconstruction using two $2 D$ laser range finders; SICK TIM310 and SICK LMS111. The scanners are mounted on a small field robot, one to measure the horizontal plane and one to measure the vertical plane. The readings from the first scanner were used to localize the robot and to build a $2 D$ map of the area, while the second scanner was used to take readings in vertical intersections. Both were then joined to form a $3 D$ scan of the environment. The reconstruction was evaluated in an artificial environment with an error of $0.7 \mathrm{~m} \pm 1.9 \mathrm{~m}$ for length, $0.005 \mathrm{~m} \pm 0.04 \mathrm{~m}$ for height and $0.05 \mathrm{~m} \pm 0.04 \mathrm{~m}$ error for width of the testing environment.

Key words: SLAM, $3 D$ reconstruction, mapping, lidar
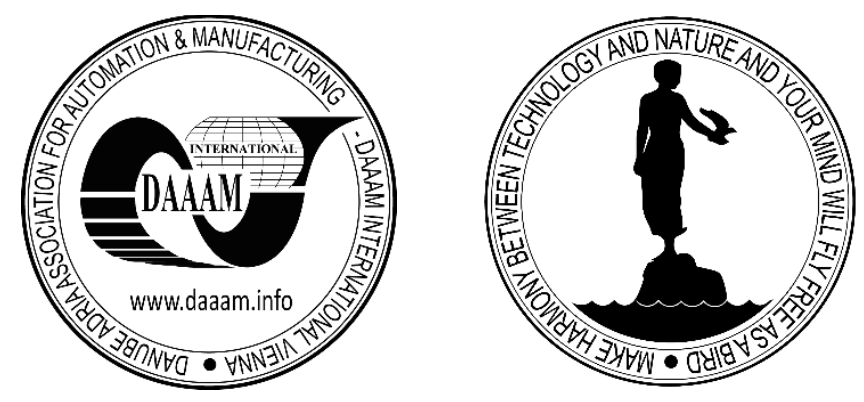

Authors' data: Lepej, P[eter]*; Assoc. Prof. Lakota, M[iran] ${ }^{* *}$; Assist. Prof. Rakun, J[urij]**; *VISTION d.o.o., Vojkova 63, Ljubljana, Slovenia, **University of Maribor, Faculty of Agriculture and Life Sciences, Pivola 10, SI-2311, Hoce, Slovenia, peter.lepej@vistion.si,miran.lakota@um.si,jurij.rakun@um.si

This Publication has to be referred as: Lepej, P[eter]; Lakota, M[iran] \& Rakun, J[urij] (2016). Simultanious Localization, Maping and Scene Reconstruction, Chapter 07 in DAAAM International Scientific Book 2016, pp.069-076, B. Katalinic (Ed.), Published by DAAAM International, ISBN 978-3-902734-09-9, ISSN 1726-9687, Vienna, Austria

DOI: $10.2507 /$ daaam.scibook.2016.07 
Lepej, P.; Lakota, M. \& Rakun, J.: Simultanious Localization, Maping and Scene R...

\section{Introduction}

Whenever a robot is placed in an unknown environment it has to localize itself if a path is to be planned or if it will build a map of the environment. But to do so, it first has to see the environment. This can be accomplished by using simple IR or ultrasonic sensors that produce trivial readings, or more complex sensors such as cameras, stereo cameras, depth cameras, single or multi-channel laser range finder (LRF) that produce more complex readings, needed for more precise applications.

If we know that the robot will only face obstacles that are on the ground, some simplifications can be taken into account and use ordinary 2D LRF and just drive between the obstacles. But if the object can hang or partly obstruct the robot movement a $3 \mathrm{D}$ reconstruction of the scene is usually the best choice when moving through an unfamiliar territory. For instance, stereo vision (Ben-Tzvi et al, 2010) uses two cameras where depth information can be extracted by analysing the same, corresponding pixel pares. The next approach uses a digital camera and a projector that projects structured light on to the scene (Jung et al, 2014), where depth information can once more be extracted by analysing the intensity of the light. The closer the object is, the brighter the light is. The final, the most expensive, are the 3D LRF (Roh et al, 2013) that use laser light to evaluate the distance to a point of the scene. In contrast to the older models the newer ones have multiple channel, which means they can measure in 3D, of course with limited resolution by the number of channels they can use.

An example use of such a 3D LRF could be to enable a field robot to navigate (Roh et al, 2013). A robot placed inside a filed with crop lines surrounding it from both sides and it has to detect them, so it will drive in between and not damaged them by running them over. Another useful scenario might be to use the 3D depth sensor to detect different fruits or crop and pick it (Dzitac et al, 2012). It can also be used to record the outline of the three crown silhouette (Kelc et al, 2015) in order to adjust the flow of the pesticide in the orchard (Berk et al, 2016; Stajnko et al, 2013). Where there are no trees, the valves are closed, when they are rare, the valves are partly open and when tick tree crowns are present, the valves are completely open.

In all cases localization and mapping algorithm (SLAM) must be used. Widely used algorithms for localization and mapping are for example Hector mapping (Kholbrecker et al, 2013) and Gmapping. Gmapping is a standard method described in (Grasetti et al, 2005) and (Graseti et al, 2006), which is based on Rao-Blackwellized particle filter to create a map and provide localization information. The method's purpose is to decrease the number of particles with filtering where precise calculation and exact deviation is considered. It uses latest information provided by LRS, reduces locational uncertainty and predicts robot's pose. Gmapping is optimized for long-range LRF and environments with large areas. In order to work it needs a laser scan data and for best results additional odometry is required. Another approach, which does not depaned on the odometry, was described by Lepej et al (2016) and uses image registration techniques to map the and localize the robot in more complex environment.

In this work we investigate the possibility to use two cheap 2D LRF to reconstruct a 3D scene instead of using a more expensive 3D LRF, limited by the number of channels. To test the approach a small field robot was fitted with two mounted LRF 
scanners, presented in the second section. The tests were conducted in indoor, artificial environment, as described in the third section. Section four concludes the paper with the most important findings and suggests possible feature improvements.

\section{Materials and methods}

\subsection{Mobile platform - Cornstar robot}

The testing mobile platform was a small field robot that is built of four major components: mechanics, actuators, embedded electronics and sensors. The mechanical part includes a four-wheel drive, two axles that can be turned individual by servomotors, automatic gearbox with forward and backward two-speed transition and a three-phase motor. The actuator part includes a pressured reservoir, two electromagnetic valves and nozzles that enable the robot to spray. The on-board digital camera and two LRFs make up the sensory part of the robot. The last but not least is the electronic part of the robot, which includes an embedded computer, peripheral circuit and a power supply. Fig. 1 depicts the image of the robot with the mounted LRF scanners.

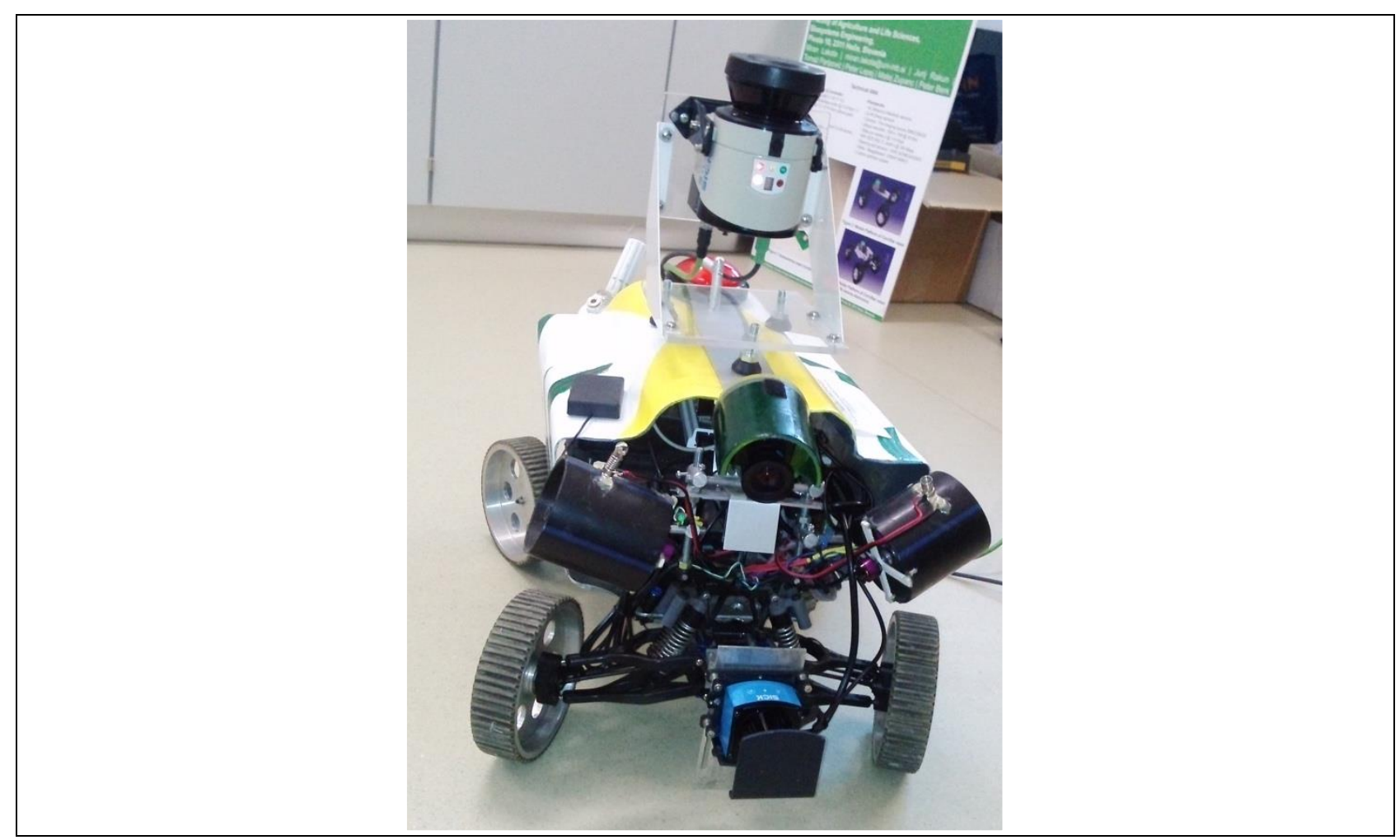

Fig. 1. Cornstar robot with two mounted LRFs; SICK TIM 310 for vertical scans and SICK LMS111 for horizontal scans

The mechanical part of the robot was built as a four-wheel drive that enables the robot to move around on the rough terrain. It is driven by a high-performance brushless motor X-power eco A4130-06BL, controlled by X-power professional 70-3P BEC controller. The motor and the controlled are powered by a two cell LiPo battery pack with $7.4 \mathrm{~V}$ voltage and a $5000 \mathrm{mAh}$ capacity. The speed of the motor is $510 \mathrm{rpm} / \mathrm{V}$ (without gear) which drives the robot with a top speed of $2 \mathrm{~m} / \mathrm{s}$ (trough gear). The two 
axles, that can be turned separately, make possible to turn the robot in the next crop line even if the space is limited at the end of the field.

Two electromagnetic valves, nozzles and a reservoir, coupled with PVC pipes, offer a possibility to spray the plants at selected locations. When plants are not affected by disease or they are simply missing from the crop line, the valves are shut and they open when the robot identifies and passes a possible area that needs to be sprayed.

The sensors on the robot include an industrial camera (The imaging source's DBK31BU03) and a two LRFs (SICK TIM 310 and SICK LMS111). The camera captures Bayer encoded images with resolution of $1024 \times 768$ pixels at $30 \mathrm{fps}$. The TIM 310 captures distances between a sensor and an obstacle in a $270^{\circ}$ radius from 0.05 up to $4 \mathrm{~m}$ distance, while the LMS111 has a sensing range from 0.4 to $18 \mathrm{~m}$. The sampling frequency of the first LRF is $15 \mathrm{~Hz}$ with $1^{\circ}$ resolution and $50 \mathrm{~Hz}$ with $0.5^{\circ}$ for the second.

The electronic part of the robot is made up of an embedded computer (Nitrogen6x) and a peripheral expansion board build around a AVR ATmega 128 microcontroller. The computer is based on AVR Cortex A9 quad core processor running at $1 \mathrm{GHz}$, with $1 \mathrm{~GB}$ of memory and can reach a performance of 2000 BogoMIPS per core. It offers a vast number of ports, where USB was used for camera and laser range sensors, while the UART port is used to communicate with the expansion board. An Ubuntu Linux (Linaro) distribution was selected for the operating system that was uploaded to a SD card, where a custom version of the kernel had to be compiled to support the USB based camera.

\subsection{Building model of the environment}

The readings from the LRFs are represented using a polar coordinate system. To get a Cartesian coordinates the following equation is applied:

$$
(x, y)=(r \cos \theta, r \sin \theta)
$$

where $\mathrm{r}$ is the distance from the LRF to a point in space (obstacle), $\theta$ is the temporary angle, while $x$ and $y$ are the final coordinates. This way the coordinates for all the reading are known but are misaligned, because both LRFs are not in one point in space and use their own coordinate systems. To aligned them Eq. (2) is applied:

$$
\left[\begin{array}{c}
x^{\prime} \\
y^{\prime} \\
z^{\prime} \\
1
\end{array}\right]=\mathbf{R}_{x} \mathbf{R}_{y} \mathbf{R}_{z} \mathbf{T}\left[\begin{array}{c}
x \\
y \\
z \\
1
\end{array}\right]
$$

where $\mathrm{x}, \mathrm{y}$ and $\mathrm{z}$ are the original coordinates, $\mathbf{T}$ is a translation matrix, $\mathbf{R}_{\mathbf{x}}, \mathbf{R}_{\mathbf{y}}$ and $\mathbf{R}_{\mathbf{z}}$ rotation matrixes along the three axis and $\mathrm{x}^{\prime}, \mathrm{y}^{\prime}$ and $\mathrm{z}$ ' are the final coordinates.

The reconstruction of a new environment consists of two parts. The first part represents the mapping algorithm (SLAM) that builds a 2D map of the environment. It is followed by the second step, point cloud assembler, which adds additional dimension to the first part. 
The SLAM algorithm utilizes readings from the horizontally mounted LRF to build a 2D map of the environment (Kohlbrecker et al, 2013; Grasetti et al, 2006; Lepej et al, 2016). For this initial experiment Hector Mapping from Darmstadt (Grasetti et al, 2006) was chosen, based on the reports for its performance in artificial, indoor environments. Besides the map the algorithm provides an estimate of current position in the environment, relative to the position of the LRF. From a given pose a static transformation can be made relative to the second vertical LRF, to construct a 3D model. To do so, the scans from multiple positions are captured and mapped to a previous recorded (SLAM) point in space. This way a 3D model of the environment is built for any environment the platform is moving. To construct a 3D model assembler ROS and PCL open source software were used.

\section{Results}

In order to evaluate the approach a test was conducted in an artificial, indoor environment (corridor), with an intent to compare the measurements of the reconstructed scene with real values, but without the interference of other factors that might occur outside (wind, sun, etc.). Figs. 2 and 3 depict a picture of a corridor in real world as well as its 3D reconstruction, while Fig. 4 depicts two close-ups from the left and right view with clearly seen objects (doors, heating radiators, ...).

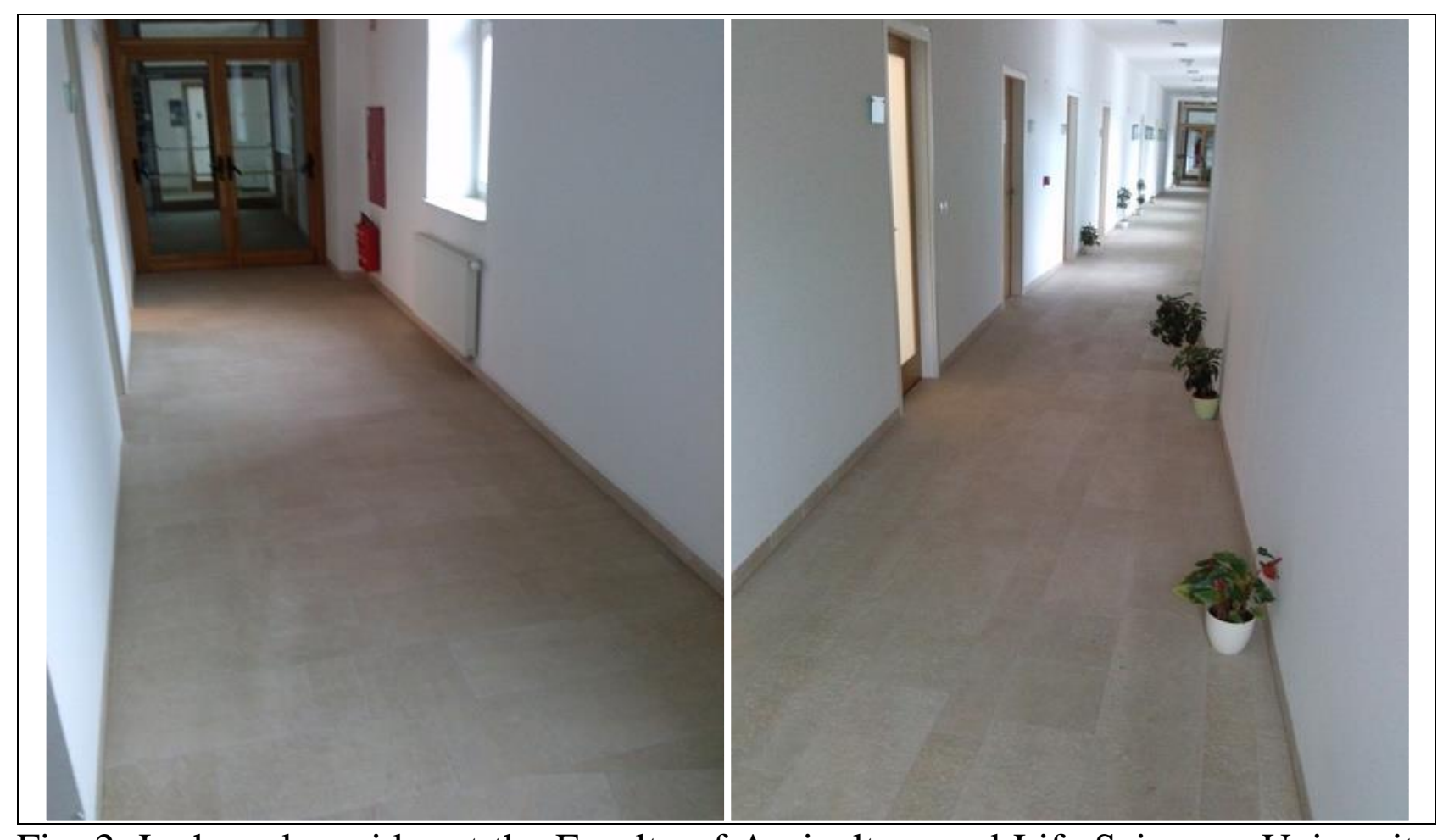

Fig. 2. L-shaped corridor at the Faculty of Agriculture and Life Sciences, University of Maribor. The left image depicts a short part, while right image depicts a long part of the corridor 
Lepej, P.; Lakota, M. \& Rakun, J.: Simultanious Localization, Maping and Scene R...

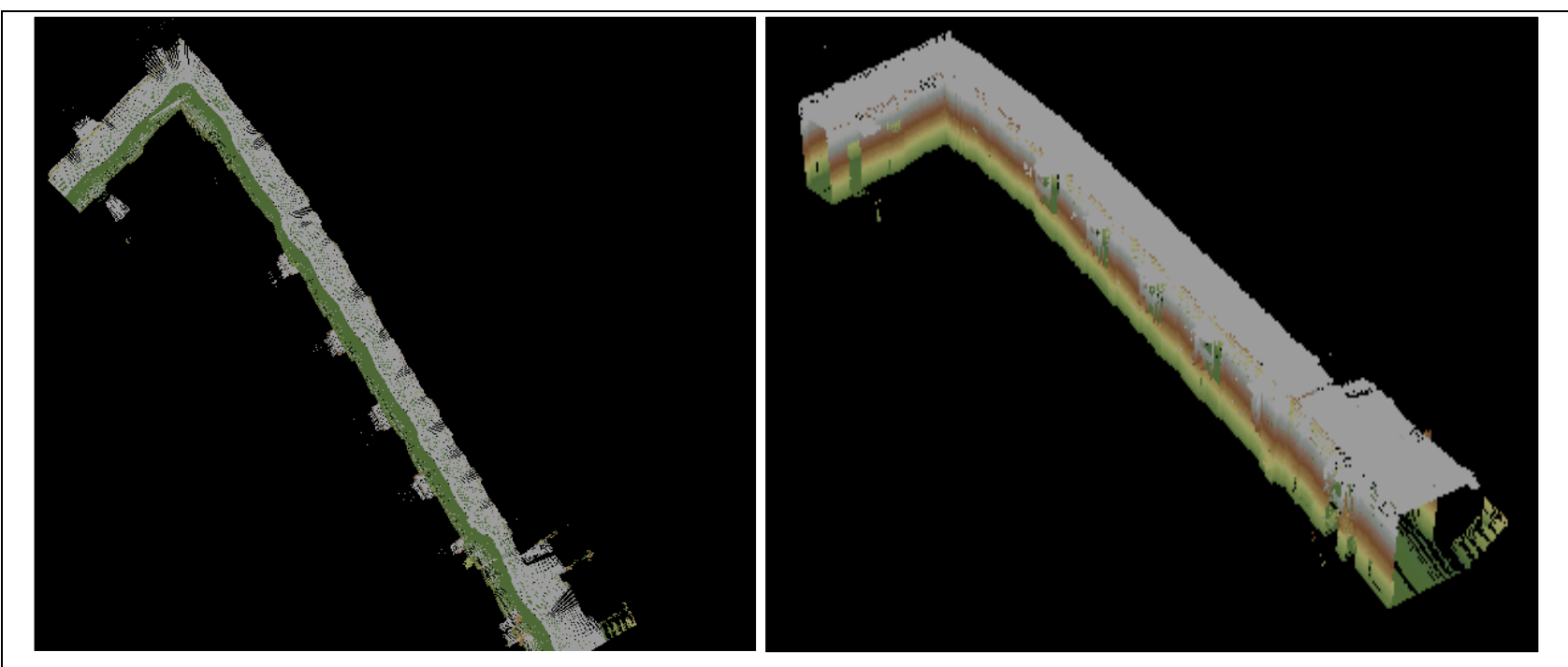

Fig. 3. The reconstructed map of the corridor. The left image depicts a bird-eye view of the corridor and the right one its $3 \mathrm{D}$ model

Fig. 4 clearly depicts objects from the scene, like doors (on the left), windows and radiators (on the right). But to verify the approach, the width and length of the reconstructed scene were measured and summarized in Tab. 1 . The measurements were first done for the short part of the corridor and then for the longer part.

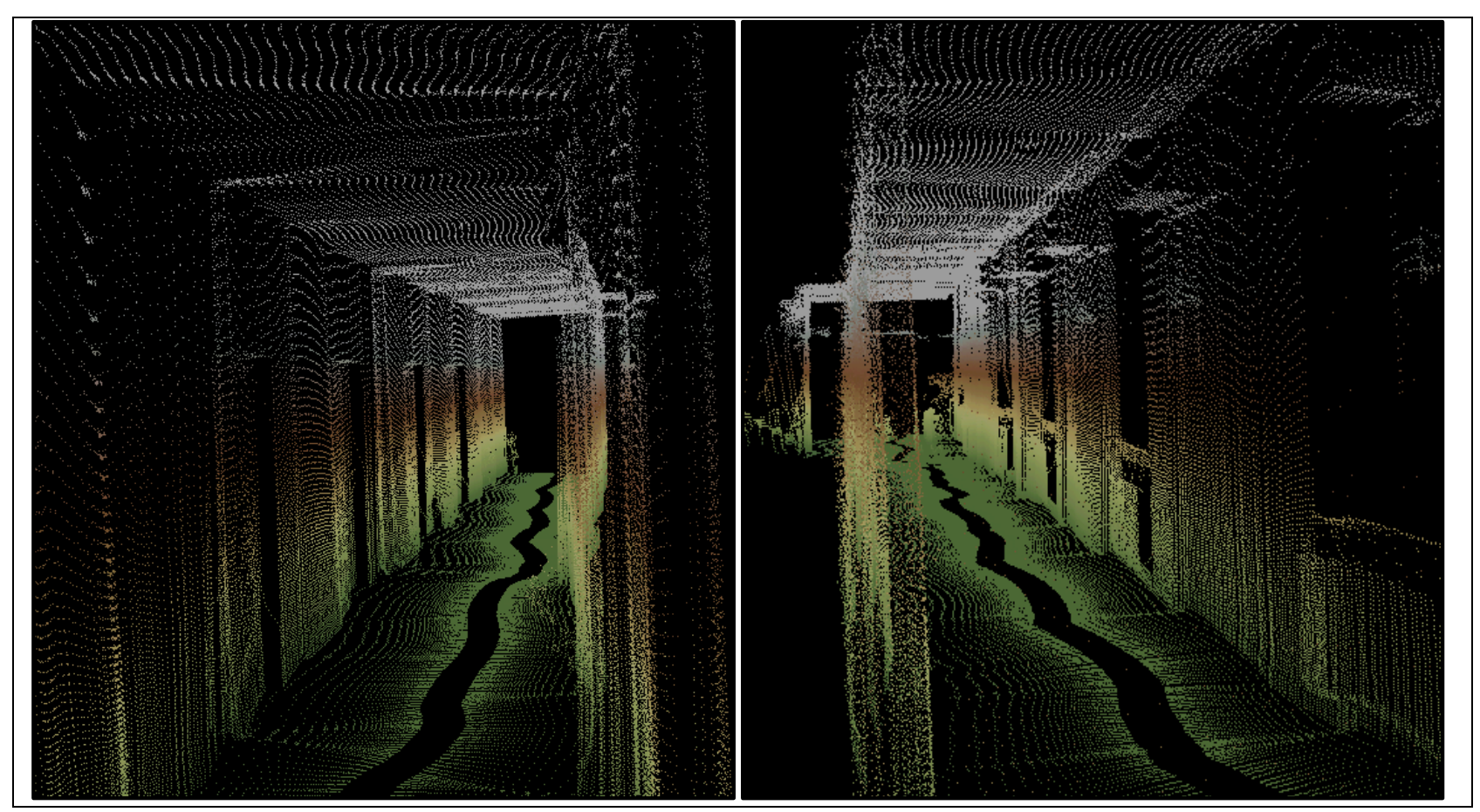

Fig. 4. Two close-ups of the longer part of the corridor (for left and right side) 


\begin{tabular}{|c|c|c|c|c|}
\hline DAAAM INT & ERNATIONAL & SCIENTIFIC BOOK & pp. 069-076 & Chapter 07 \\
\hline Part & Description & Real values [m] & Reconstructed values [m] & Error $[\mathrm{m}]$ \\
\hline & Width 1 & 2.2 & 2.28 & 0.08 \\
\hline$\overparen{D}=$ & Width 2 & 2.2 & 2.2 & 0 \\
\hline \pm 0 & Height 2 & 2.98 & 3 & -0.02 \\
\hline$\varpi$ 氙 & Length 1 & 9.4 & 9 & -0.4 \\
\hline & Width 3 & 1.93 & 2 & 0.07 \\
\hline 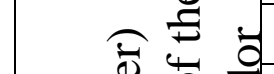 & Width 4 & 3.1 & 3.06 & 0.04 \\
\hline$=0$ & Height 4 & 2.98 & 3.01 & 0.03 \\
\hline 를 & Length 2 & 39.98 & 34.64 & -5.34 \\
\hline & & & Average: & $0.7 \pm 1.9$ \\
\hline
\end{tabular}

Tab. 1. Eight different measurements for two parts of the indoor scene.

The overall average error from Tab. 1 is $0.7 \mathrm{~m} \pm 1.9 \mathrm{~m}$, but separate errors for width, length and height should be considered. The average width error is $0.05 \mathrm{~m} \pm$ $0.04 \mathrm{~m}$, average length error $-2.87 \mathrm{~m} \pm 3.49 \mathrm{~m}$ and average height error $0.005 \mathrm{~m} \pm 0.04$ $\mathrm{m}$.

\section{Conclusion}

This paper describes an experimental approach to build a 3D model of the scene by using two 2D LRFs, instead of using one 3D expensive sensor with limited number of channels. The approach was developed and tested on a small field robot, but with a system that could be mounted on bigger version or any kind of agricultural machine. In contrast to true 3D LRFs it is not able to observe the environment from distance, but it has to pass through in order to produce a 3D reconstruction. On the other hand, it offers a higher degree of resolution with lower costs in comparison to 3D LRFs.

The results from indoor tests reached an average error of $0.7 \mathrm{~m} \pm 1.9 \mathrm{~m}$, caused almost by the error in length, scanned by SICK LMS111 LRF. The length of the map is constructed by Hector SLAM algorithm, which caused $5.34 \mathrm{~m}$ error in $39.98 \mathrm{~m}$ long corridor. The scans that recorded height and width of the corridor reached $0.005 \mathrm{~m} \pm$ $0.04 \mathrm{~m}$ and $0.05 \mathrm{~m} \pm 0.04 \mathrm{~m}$ error, respectively.

For further research it would be suggested to use two LMS111 LRFs as it works faster, more accurately and with higher resolution. Its only limit is the starting distance of $0.4 \mathrm{~m}$, which should not be a problem to most agricultural scenarios. The second suggestion is to use a different SLAM algorithm that would improve the accuracy of 2D map and 3D scene reconstruction. 
Lepej, P.; Lakota, M. \& Rakun, J.: Simultanious Localization, Maping and Scene R...

\section{References}

Ben-Tzvi, P. \& Xu, X. (2010). An embedded feature-based stereo vision system for autonomous mobile robots, Workshop on Robotic and Sensors Environments (ROSE), 2010 IEEE International, pp. $1-6$

Berk, P.; Hocevar, M.; Stajnko, D. \& Belsak, A. (2016). Development of alternative plant protection product application techniques in orchards, based on measurement sensing systems: A review, Computers and electronics in agriculture, vol. 124, pp. 273-288

Dzitac, P. \& Mazid, A. M. (2012). A depth sensor to control pick-and-place robots for fruit packaging, 12th International Conference on Control Automation Robotics \& Vision (ICARCV), pp. 949 - 954

Grisetti, G.; Stachniss, C. \& Burgard, W. (2005). Improving Grid-based SLAM with Rao Blackwellized Particle Filters by Adaptive Proposals and Selective Resampling, Proceedings of the IEEE International Conference on Robotics and Automation.

Grisetti, G.; Stachniss, C. \& Burgard, W. (2006). Improved Techniques for Grid Mapping with Rao-Blackwellized Particle Filters, IEEE Transactions in Robotics, Volume 23, pp. 34-46

Jung, H. \& Lyou, J. (2014). 3D map building using the kinect mounted on a mobile robot, IEEE International Conference on Industrial Technology (ICIT), pp. $604-608$. Kelc, D.; Vindiš, P. \& Lakota, M. (2015). Measurements of photosynthesis and transpiration of apple trees. V: KATALINIĆ, Branko (ur.). DAAAM International scientific book 2015, (DAAAM International scientific book, ISSN 1726-9687). Vienna: DAAAM International Vienna, pp. 199-208

Kohlbrecher, S.; Meyer, J.; Graber, T.; Petersen, K.; Klingauf, U. \& Stryk, O. (2013). Hector Open Source Modules for Autonomous Mapping and Navigation with Rescue Robots, Department of Computer Science, TU Darmstadt, Germany

Lepej, P. \& Rakun, J. (2016). Simultaneous localisation and mapping in a complex field environment, Biosystems Engineering, Volume 150, pp 160-169

Roh, H. C.; Sung, C. H. \& Chung, M. J. (2013). Rapid SLAM using simple map representation in indoor environment, 19th Korea-Japan Joint Workshop on Frontiers of Computer Vision, pp. 225 - 229

Stajnko, D.; Vindiš, P. \& Lakota, M. (2013). Newel approaches for targeted spraying in orchards. V: KATALINIĆ, Branko (ur.), TEKIC, Zeljko (ur.). DAAAM International scientific book 2013, (DAAAM International scientific book, ISSN 17269687). Vienna: DAAAM International Publishing, pp. 561-570 